\title{
Hadron production in lepton-nuclei interactions at high energies: Monte Carlo generator HARDPING 2.0
}

\author{
Ya. A. Berdnikov ${ }^{\& \%}$, A. E. Ivanov ${ }^{\& \% *}$ V. T. Kim\&\%, V. A. Murzin $\%$ \\ ${ }^{\&}$ St. Petersburg State Polytechnical University, 195251 St. Petersburg, Russia \\ ${ }^{\%}$ St. Petersburg Nuclear Physics Institute NRC KI, 188300 Gatchina, Russia
}

December 14, 2018

\begin{abstract}
Hadron production in lepton-nucleus interactions at high-energies is considered in framework of developing Monte Carlo event generator HARDPING (HARD Probe INteraction Generator). Such effects as formation length, energy loss and multiple rescattering for produced hadrons and their constituents are implemented into the HARDPING 2.0. Available data from HERMES collaboration on hadron production in lepton-nucleus collisions are described by the present version of the HARDPING generator in a reasonable agreement.
\end{abstract}

Hadronisation of quarks and gluons is one of the most intriguing parts of nonperturbative QCD. Use of nuclear targets may allow to reveal important features of space-time picture of hadronisation, like hadron formation length and energy loss, see, e.g, for a review [1, 2] and references therein. The understanding of quark propagation in nuclear medium is crucial for the interpretation of ultrarelativistic heavy ion collisions, as well as high energy protonnucleus and lepton-nucleus interactions. To simplify interpretation of observable effects one can consider at the beginning hadron production in lepton scattering off nuclei. In case of deep inelastic scattering of lepton on nucleus there can be two stages of hadronisation. The first stage is predominantly perturbative. At this stage after hard scattering a struck a struck quark propagates through the nuclear medium being in point-like parton state experiencing a little attenuation only. This effect is known as Landau-Pomeranchuck-Migdal effect in QCD [3, 4, [5, 6, 7, 8, 9, 10]. At the end of the first stage, a pre-hadron state (a color dipole or constituent quark) is formed [11, 12, 13. In the second stage pre-hadron state with smaller than hadron cross section interact with nuclear medium. There is finally formed hadron at the second stage. At the large enough energies of produced hadrons the nonperturbative stage of hadron formation is evolving beyond the nucleus [22].

*e-mail: ivanovae@pnpi.spb.ru 
The aim of the work is to study these effects for case of lepton-nuclei collisions using a developing Monte Carlo (MC) event generator. The generator HARDPING (HARD Probe INteraction Generator) is based on MC generators PYTHIA [14] and HIJING [15. The first version of HARDPING describes experimental data on Drell-Yan reaction off nuclei reasonably well [16, 17]. It takes into account the effects related with interaction of projectile hadron and its constituents in nuclear matter before hard scattering for lepton-pair production off nuclei. The second version of HARDPING, presented here, describes, in addition, hadron production in lepton-nuclei interactions. It incorporates the following effects: formation length, energy loss and multiple soft rescatterings.

The experimental results on semi-inclusive leptoproduction of hadrons off nuclei [18, 19] are presented in terms of hadron multiplicity ratios $R_{M}^{h}$ with nuclear $(A)$ and deuteron $(D)$ targets, as functions of virtual photon energy $(\nu)$, its fraction taken by hadron $\left(z_{h}\right)$ and hadron transverse momentum squared $p_{\perp}^{2}$ :

$$
R_{M}^{h}(x)=\frac{1}{N_{A}^{D I S}} \frac{d N_{A}^{h}}{d x} / \frac{1}{N_{D}^{D I S}} \frac{d N_{A}^{h}}{d x}
$$

where $N_{A}^{D I S}$ and $N_{D}^{D I S}$ are yelds of inclusive deep-inelastic scattering leptons on nuclei $A$ and $D, \frac{d N_{A}^{h}}{d x_{2}}$ and $\frac{d N_{D}^{h}}{d x}$ are yields of semi-inclusive hadrons as a function of $x$, here $x$ is either $z_{h}$ or $p \perp^{2}$. In absence of nuclear effects, the ratio $R_{M}^{h}$ should be equal to 1 . The experimental results show that this is the case at high transferred energy $\nu$ [18].

It is well established from theoretical and experimental studies of hadron-nucleus collisions at high energy that hadrons are not produced at the point of collision but only after some "formation" length [1]. In the Lund string fragmentation model, the production of hadrons is described as two stage process. At the first perturbative stage a pre-hadron at the end of the string is formed. On the next nonperturbative stage a hadron is formed. Before a pre-hadron is formed, the struck quark propagates through the nuclear matter with a very small cross section (in this work we neglect it). It takes some time at the perturbative stage to form a pre-hadron (formation time, $t_{p}$ or formation length, $l_{p}$ ). When the pre-hadron is formed, it interact with nuclear matter via pre-hadron cross section, which is different from hadronic cross section. And also it takes an extra time to form the final hadron from pre-hadron state. So, the formation length consist of two parts $\left(l_{p}\right.$ and $\left.l_{n}\right)$, corresponding the two stages of hadronisation.

There are two approaches to calculate formation length with two stages. The first is based on an oversimplified description of nonperturbative stage [1], while the second one [11] is based on Lund string model, but neglecting the energy loss effects during the perturbative stage. The present work is based on the both above approaches with including the effect of energy loss at the perturbative stage and using Lund string model at the nonperturbative stage.

In the first approach the distribution on pre-hadron formation time can be written in the following form [1]:

$$
W\left(t_{p}, z_{h}, Q^{2}, \nu\right)=N \int_{0}^{1} \frac{d \alpha}{\alpha} \delta\left[z_{h}-\left(1-\frac{\alpha}{2}\right) \frac{E_{q}\left(t_{p}\right)}{\nu}\right] \times
$$




$$
\begin{gathered}
\int_{\Lambda_{Q C D}^{2}}^{Q^{2}} \frac{d k_{\perp}^{2}}{k_{\perp}^{2}} \delta\left[k_{\perp}^{2}-\frac{2 \nu}{t_{p}} \alpha(1-\alpha)\right] \int d l_{\perp}^{2} \delta\left[l_{\perp}^{2}-\frac{9}{16} k_{\perp}^{2}\right] \times \\
\quad \int_{0}^{1} d \beta \delta\left[\beta-\frac{\alpha}{2-\alpha}\right]\left|\Psi_{h}\left(\beta, l_{\perp}\right)\right|^{2} S\left(t_{p}, z_{h}, Q^{2}, \nu\right)
\end{gathered}
$$

here $t_{p}$ is pre-hadron formation time, $z_{h}$ is fraction of virtual photon energy carried out by the hadron, $Q^{2}$ is virtual photon virtuality, $\nu$ is virtual photon energy, $\Lambda_{Q C D}$ is $\mathrm{QCD}$ constant, $\Psi_{h}\left(\beta, l_{\perp}\right)$ meson wave function, $E_{q}\left(t_{p}\right)=\nu-\Delta E\left(t_{p}\right)$ is quark energy and $\Delta E\left(t_{p}\right)$ is quark energy losses due to perturbation gluon radiation, $\delta$ is delta-function, $S\left(t_{p}, z_{h}, Q^{2}, \nu\right)$ is Sudakov suppression factor. $\Delta E\left(t_{p}\right)$ can be taken in the following form:

$$
\begin{gathered}
\Delta E(t)=\nu \int_{\Lambda_{Q C D}^{2}}^{Q^{2}} d k_{\perp}^{2} \frac{4 \alpha_{s}\left(k_{\perp}^{2}\right)}{3 \pi} \int_{0}^{1} d \alpha \frac{1}{k_{\perp}^{2}} \times \\
\theta\left(t-\frac{2 \nu \alpha(1-\alpha)}{k_{\perp}^{2}}\right) \theta\left(1-z_{h}-\alpha\right)
\end{gathered}
$$

where $\theta$ is step-function, $\alpha_{S}$ is strong coupling. This approach works only for leading hadrons with $z_{h}>0.5$, (see Fig, 1, the dashed line), which is not suitable for full MC simulation.
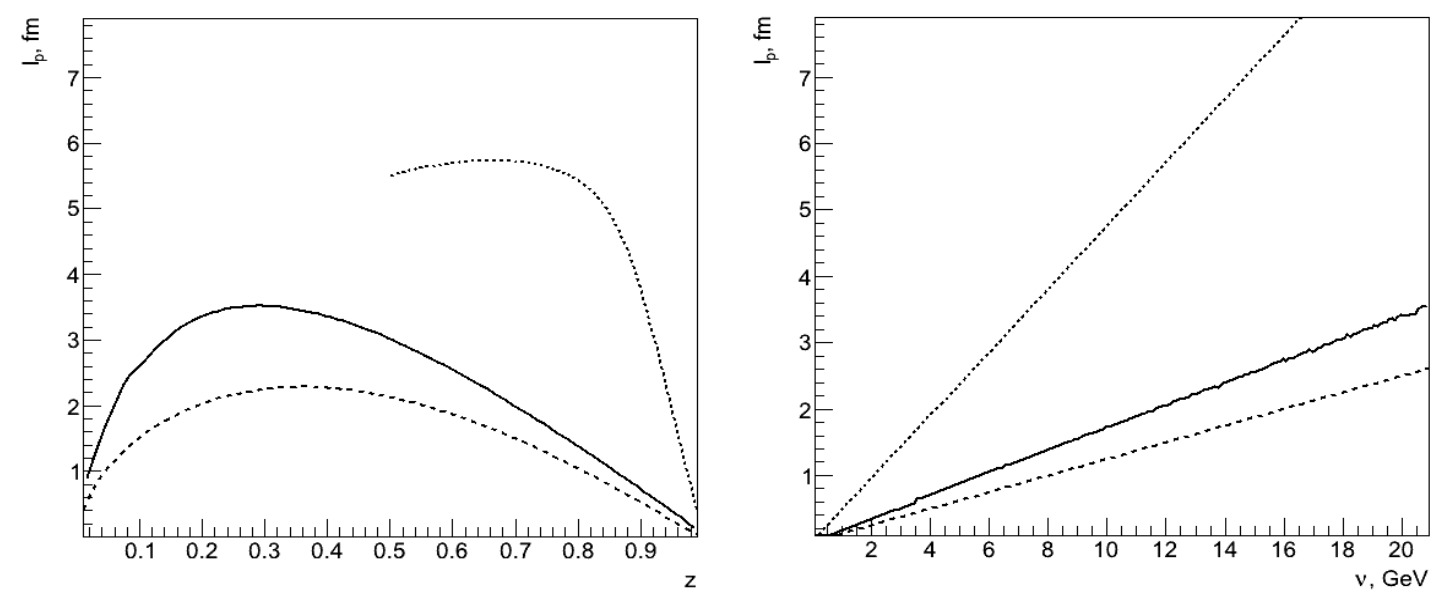

Figure 1: Formation length as a function of $z_{h}$ and as a function of $\nu$. The dotted lines correspond to the first approach (B.Z. Kopeliovich et al. [1]) for $z_{h}>0.5$, the dashed lines corresponds to the second approach (A. Accardi et al. [11]) and the solid lines corresponds to HARDPING 2.0 calculations

The second approach is based on Lund string model for nonperturbative hadronisation neglecting its perturbative stage [11. In this approach probability to have pre-hadron formation length $l_{p}$ can be written in the following form:

$$
\begin{gathered}
P\left(l_{p} ; z_{h}, L\right)=\frac{z_{h} L}{l_{p}-z_{h} L}\left[\frac{l_{p}}{\left(l_{p}+z_{h} L\right)\left(1-z_{h}\right)}\right]^{C} \times \\
\left(\delta\left[l_{p}-\left(1-z_{h}\right) L\right]+\frac{1+C}{l_{p}-z_{h} L} \theta\left[\left(1-z_{h}\right) L-l_{p}\right]\right) \\
\times \theta\left[l_{p}\right] .
\end{gathered}
$$


where parameter $C=0.3$ [11], and parameters $k$ and $L$ are string tension and ratio of the virtual photon energy to string tension $L=\nu / k$.

In the presented here approach the effect of energy loss was incorporated into the HARDPING 2.0 using PYTHIA MC implementation of parton shower for the perturbative stage and Lund string model for the nonperturbative one. On the Fig.1 the dependences of formation length on $z_{h}$ and $\nu$ are plotted, the dotted lines - the first approach [1], the dashed lines - the second approach [11], the solid lines - HARDPING 2.0 simulation.

During the perturbative stage, corresponding to the formation length $l_{p}$, a constituent quark (or pre-hadron) state is formed. It can interact with intranuclear nucleons via inelastic pre-hadronic cross-section (or inelastic quark-nucleon cross-section). At the end of the nonperturbative stage the observed hadron is formed.

Produced pre-hadrons and hadrons can undergo soft collisions with intranuclear nucleons (with small momentum transfers: $|t|<1 \mathrm{GeV}^{2}$ ). So, one has to take into account their soft multiple rescattering.

The transverse momentum distribution of constituent quarks after one soft interaction can be parameterised in the following form [20, 16, 17]:

$$
f_{p}\left(\overrightarrow{p_{\perp}}\right)=\frac{B^{2}}{2 \pi} e^{-B p_{\perp}}
$$

where $B=\frac{2}{\left\langle k_{p}\right\rangle}$, where $\left\langle k_{p}\right\rangle$ is mean value of quark transverse momentum. $f_{p}\left(\overrightarrow{p_{\perp}}\right)$ is a differential distribution of quark in quark-nucleon interaction normalised on unity.

Probability to have no interactions between the points with coordinates $(z, \vec{b})$ and $(z+\lambda, \vec{b})$ can be written in the next form:

$$
P(\lambda ; z, \vec{b})=e^{-\sigma T(\vec{b}, z, \lambda)}
$$

where $T(\vec{b}, z, \lambda)$ is:

$$
T(\vec{b}, z, \lambda)=(A-1) \int_{z}^{z+\lambda} \rho\left(\vec{b}, z^{\prime}\right) d z^{\prime}
$$

$\rho(\vec{b}, z)$ is nuclear density and $\sigma$ is quark-nucleon (pre-hadron-nucleon) or hadron-nucleon inelastic cross section.

Simulations of lepton-nuclei collisions obtained by HARDPING 2.0 were compared with HERMES data [18, 19]. The results are shown on Figs. 2,[3,

The performed simulations shown a reasonable agreement of MC model HARDPING 2.0 with the experimental HERMES data [18, 19]. This allowed to fix model parameters such as inelastic quark-nucleon (pre-hadron-nucleon) cross-section $\sigma=10 \mathrm{mb}$ and string tension $k=1.7 \mathrm{GeV} / \mathrm{Fm}$. Comparision with EMC [21] and SLAC [22, 23] data shown also a good agreement and it will be presented elsewhere.

To summarise, the effects of the two-stage hadronisation and multiple soft interactions inside of nucleus for produced hadrons and their constituents were implemented into MC generator HARDPING 2.0. The developed MC generator HARDPING 2.0 is allowed to describe reasonably well the HERMES data [18, 19] on hadron production in positron-nucleus scattering at $27.6 \mathrm{GeV}$. 

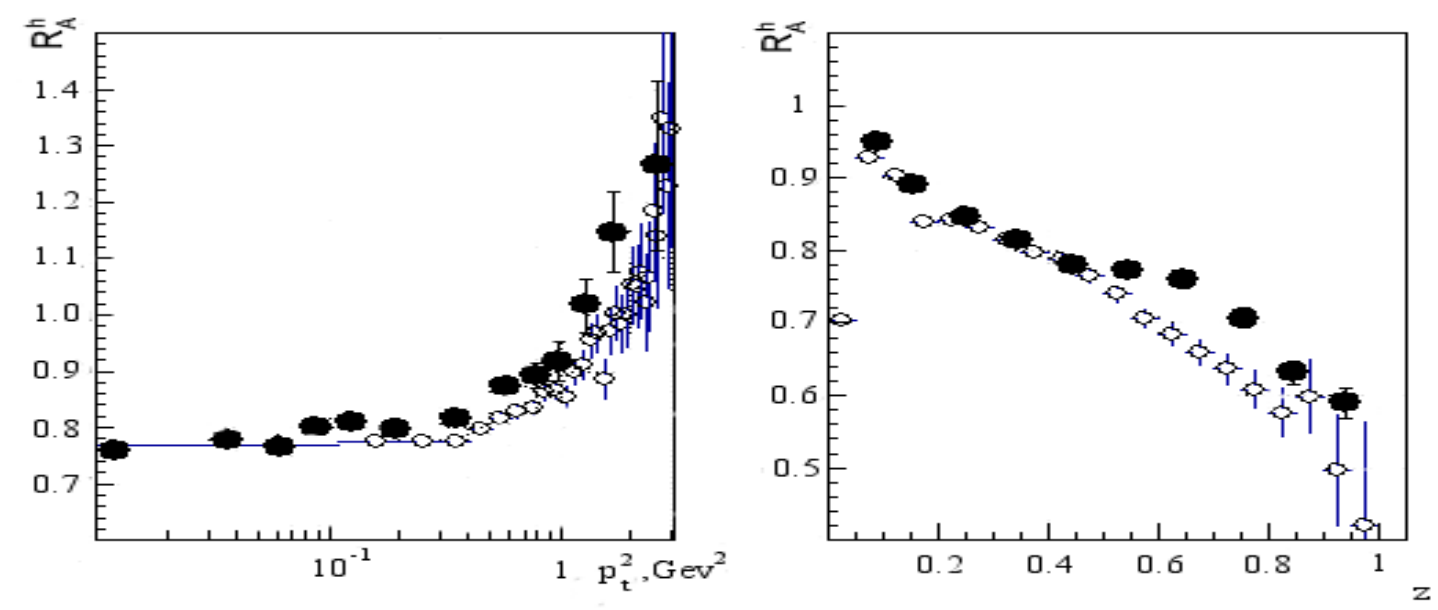

Figure 2: Multiplicity ratio $\left(R_{M}^{h}\right)$ of charged hadrons for krypton $(K r)$ and deuteron $(D)$ targets as a function of $p_{\perp}^{2}$ and as a function of $z_{h}$ at positron beam energy $27.6 \mathrm{GeV}$. The solid points correspond to HERMES data [18] and the open points are obtained by HARDPING 2.0
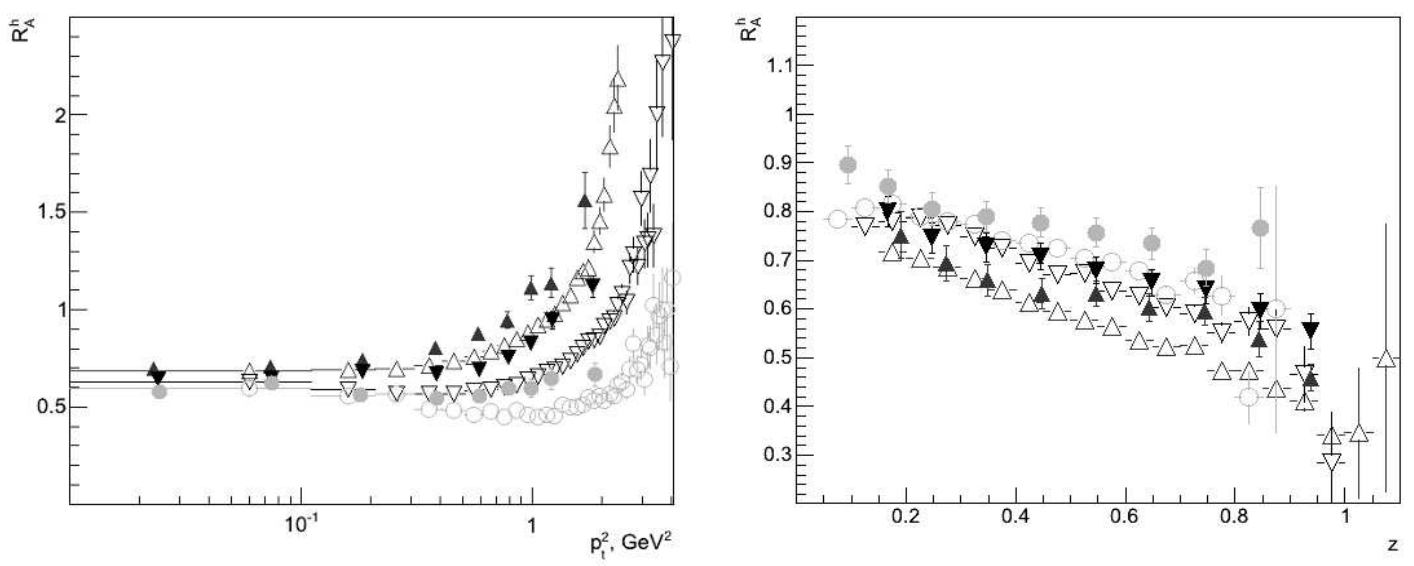

Figure 3: Multiplicity ratio $\left(R_{M}^{h}\right)$ of $\pi^{+}$-mesons for xenon $(X e)$ and deuteron $(D)$ targets as a function of $p_{\perp}^{2}$ for different $z_{h}$ values and as a function of $z_{h}$ for different $\nu$ values at positron beam energy 27.6 GeV. The solid points correspond to HERMES data [19] and the open points are obtained by HARDPING 2.0

The authors thank S.L. Belostotsky for useful discussions on the HERMES data. This work was supported in parts by the Ministry of Education of the Russian Federation, under the contract No. 02.740.11.0572 of the Federal task program "Research and educational community of innovative Russia" for 2009-2013 and by the RF President grant NS-3383.2010.2. 


\section{References}

[1] B. Z. Kopeliovich, J. Nemchik, E. Predazzi, and A. Hayashigaki, Nucl. Phys. A 740, 211 (2004) hep-ph/0311220].

[2] R. Baier, D. Schiff and B. G. Zakharov, Ann. Rev. Nucl. Part. Sci. 50, 37 (2000) hep-ph/0002198.

[3] R. Baier, Y.L. Dokshitzer, A.H. Mueller et al., Nucl. Phys. B 484, 265 (1997) hep-ph/9608322.

[4] B. G. Zakharov, JETP Lett. 65, 615 (1997) [Pis'ma Zh. Eksp. Teor. Fiz. 65, 585 (1997)] hep-ph/9704255.

[5] E. Levin, Phys. Lett. B 380, 399 (1996) hep-ph/9508414.

[6] U. A. Wiedemann, Nucl. Phys. B 588, 303 (2000) hep-ph/0005129.

[7] X. N. Wang and X. F. Guo, Nucl. Phys. A 696, 788 (2001) hep-ph/0102230.

[8] M. Gyulassy, P. Levai and I. Vitev, Nucl. Phys. B 594, 371 (2001) nucl-th/0006010.

[9] P. B. Arnold, G. D. Moore and L. G. Yaffe, JHEP 0206, 030 (2002) hep-ph/0204343.

[10] K. Zapp, J. Stachel and U.A. Wiedemann, Phys. Rev. Lett. 103, 152302 (2009) arXiv:0812.3888 [hep-ph]].

[11] A. Accardi, V. Muccifora and H. J. Pirner, Nucl. Phys. A 720, 131 (2003) nucl-th/0211011.

[12] A. Accardi, D. Grunewald, V. Muccifora and H. J. Pirner, Nucl. Phys. A 761, 67 (2005) hep-ph/0502072.

[13] S. Domdey, D. Grunewald, B. Z. Kopeliovich and H. J. Pirner, Nucl. Phys. A 825, 200 (2009) arXiv:0812.2838 [hep-ph]].

[14] T. Sjostrand, S. Mrenna and P. Z. Skands, JHEP 0605, 026 (2006) hep-ph/0603175.

[15] M. Gyulassy and X. N. Wang, Comput. Phys. Commun. 83, 307 (1994) nucl-th/9502021.

[16] Y.A. Berdnikov, V.T. Kim, V.F. Kosmach et al., Eur. Phys. J. A 26, 179 (2005) hep-ph/0510260.

[17] Y. A. Berdnikov, M. E. Zavatsky, V.T. Kim et al., Phys. Atom. Nucl. 69, 445 (2006) [Yad. Fiz. 69, 467 (2006)].

[18] A. Airapetian, N. Akopov, Z. Akopov et al. [HERMES Collaboration], Phys. Lett. B 577, 37 (2003) hep-ex/0307023. 
[19] A. Airapetian, N. Akopov, Z. Akopov et al. [HERMES Collaboration], Eur. Phys. J. A 47, 113 (2011) arXiv:1107.3496 [hep-ex]].

[20] A. V. Efremov, V. T. Kim and G. I. Lykasov, Sov. J. Nucl. Phys. 44, 151 (1986) [Yad. Fiz. 44, 241 (1986)].

[21] J. Ashman, B. Badelek, G. Baum et al. [European Muon Collaboration], Z. Phys. C 52, 1 (1991).

[22] O. Benhar, S. Fantoni, G. I. Lykasov and N. V. Slavin, Phys. Rev. C 55, 244 (1997).

[23] P. V. Degtyarenko, J. Button-Shafer, L. Elouadrhiri et al., Phys. Rev. C 50, 541 (1994). 